\title{
Padrões histopatológicos das lesões descamativas e ulcerativas da pele em cães com leishmaniose ${ }^{1}$
}

\section{Histopathological pattern of exfoliative and ulcerative skin lesions in dogs with leishmaniasis}

\author{
Rafael Torres Neto ${ }^{2 *}$; Marcela M. P. Rodrigues ${ }^{3}$; Renée Laufer Amorim ${ }^{4}$; Lissandro \\ Gonçalves Conceição ${ }^{5}$ Maria Cecília Rui Luvizotto ${ }^{6}$; Sônia R. V. S.Franco ${ }^{7}$
}

\section{Resumo}

\begin{abstract}
A leishmaniose visceral canina é uma doença infecciosa crônica considerada endêmica em algumas regiões do estado de São Paulo e, apesar de ser uma enfermidade sistêmica, no cão, a maioria dos sinais clínicos são dermatológicos. Foram avaliados trinta cães com diagnóstico de leishmaniose, da região de Araçatuba, pelos exames parasitológico e sorológico. Os cães apresentando apenas sinais dermatológicos foram divididos em dois grupos: um grupo com descamação e outro com ulceração. Os padrões histopatológicos da pele encontrados nos cães com lesões descamativas $(n=15)$ foram de dermatite perianexial $(5 / 15,33,3 \%)$, dermatite perivascular superficial $(1 / 15,6,6 \%)$, dermatite nodular $(1 / 15,6,6 \%)$, e dentre as dermatites mistas $(8 / 15,53,3 \%)$, dermatite intersticial/perianexial $(1 / 8$, $12,5 \%)$, dermatite liquenóide/perivascular superficial e profunda $(1 / 8,12,5 \%)$, dermatite perivascular superficial e profunda/perianexial $(1 / 8,12,5 \%)$ e dermatite perivascular superficial/perianexial $(5 / 8$, $62,5 \%)$. Os cães com lesões ulcerativas $(\mathrm{n}=15)$ apresentaram padrões histopatológicos de dermatite perivascular superficial e profunda $(5 / 15,33,3 \%)$, dermatite difusa $(3 / 15,20 \%)$, dermatite perianexial $(2 / 15,13,3 \%)$, dermatite nodular $(1 / 15,6,6 \%)$, e dentre as dermatites mistas $(4 / 15,26,6 \%)$, dermatite intersticial/perivascular superficial e profunda $(1 / 4,25 \%)$, dermatite nodular/perianexial $(1 / 4,25 \%)$, dermatite fibrosante/perianexial $(1 / 4,25 \%)$ e dermatite perivascular superficial e profunda/perianexial $(1 / 4,25 \%)$. A presença de formas amastigotas foram observadas em oito cães $(8 / 15,53,3 \%)$ com dermatite descamativa e sete cães $(7 / 15,46,6 \%)$ com dermatite ulcerativa.

Palavras-chave: Dermatite descamativa, dermatite ulcerativa, Leishmaniose, dermatopatologia, cão
\end{abstract}

\footnotetext{
Abstract

Canine visceral leishmaniasis is an endemic infectious disease in some São Paulo state regions and even though it is a systemic disease, in the dog, the main clinical sign is dermatological. Thirty dogs with positive results in serology and parasitological exams for leishmaniasis from Araçatuba city were evaluated. They just showed dermatological signs and were divided in two groups, one with exfoliative

1 Apoio financeiro: FUNDUNESP.

2 Aluno do programa de Pós-graduação em Medicina Veterinária (área de concentração: Clínica Veterinária, nível Doutorado), UNESP, Botucatu. Distrito de Rubião Jr, s/n, 18618.000, Departamento de Clínica Veterinária - FMVZ, UNESP, Botucatu, SP. rphtorres@yahoo.com.br

3 Aluna do programa de Pós-graduação em Medicina Veterinária (área de concentração: Clínica Veterinária, nível Doutorado),

4 Professora, Doutora, Departamento de Clínica Veterinária - FMVZ, UNESP, Botucatu, SP.

5 Professor, Doutor, Departamento de Veterinária, UFV, Viçosa, MG.

6 Professora, Doutora, Departamento de Clínica, Cirurgia e Reprodução Animal da Faculdade de Medicina Veterinária, FOA, UNESP, Araçatuba, SP.

7 Professora, Doutora, Departamento de Clínica Veterinária - FMVZ, UNESP, Botucatu, SP.
} UNESP, Botucatu.

* Autor para correspondência 
$(\mathrm{n}=15)$ and other with ulcerative $(\mathrm{n}=15)$ lesions. Histopathological patterns in the group of exfoliative lesions were: periadnexial dermatitis $(5 / 15,33,3 \%)$, superficial perivascular dermatitis $(1 / 15,6,6 \%)$, nodular dermatitis $(1 / 15,6,6 \%)$ and mixed dermatitis $(8 / 15,53,3 \%)$, including intersticial/periadnexial dermatitis $(1 / 8,12,5 \%)$, lichenoid/perivascular superficial and deep dermatitis $(1 / 8,12,5 \%)$, perivascular superficial and deep/periadnexial dermatitis $(1 / 8,12,5 \%)$ and superficial perivascular/perianexial dermatitis $(5 / 8,62,5 \%)$. In the group of ulcerative lesions, the histopathological patterns were: perivascular superficial and deep dermatitis $(5 / 15,33,3 \%)$, diffuse dermatitis $(3 / 15,20 \%)$, periadnexial dermatitis $(2 / 15,13,3 \%)$, nodular dermatitis $(1 / 15,6,6 \%)$ and mixed dermatitis $(4 / 15,26,6 \%)$, including intersticial/ perivascular superficial and deep dermatitis $(1 / 4,25 \%)$, nodular/periadnexial dermatitis $(1 / 4$, $25 \%)$, fibrosing/perianexial dermatitis $(1 / 4,25 \%)$ and perivascular superficial and deep/ periadnexial dermatitis $(1 / 4,25 \%)$. Parasites were found in eight dogs $(8 / 15,53,3 \%)$ with exfoliative dematitis and seven $(7 / 15,46,6 \%)$ with ulcerative dermatitis.

Key words: Exfoliative dermatitis, ulcerative dermatitis, Leishmaniasis, dermatopathology, dog

\section{Introdução}

A leishmaniose visceral americana (LVA) é uma doença infecciosa de evolução crônica causada pelo protozoário Leishmania (L.) chagasi, transmitido, principalmente, pelo vetor Lutzomyia longipalpis, conhecido popularmente como mosquito palha, birigui ou tatuquiras. Em algumas regiões da Colômbia e Venezuela, o vetor é o Lutzomia evansi e, no Brasil, no estado do Mato Grosso, o Lutzomia cruzi também foi incriminado como vetor da doença (SANTOS et al., 1998). A espécie canina é considerada o principal reservatório doméstico, constituindo parte do ciclo epidemiológico de transmissão ao homem (LAISON; SHAW, 1987). A leishmaniose canina (LC) e a leishmaniose visceral humana são endêmicas em muitos estados do Norte, Nordeste, Centro-Oeste e Sudeste do Brasil. (EVANS et al., 1990) e um número crescente de casos tem ocorrido em algumas regiões do estado de São Paulo (FEITOSA et al., 2000).

Apesar da natureza viscerotrópica do protozoário, as lesões dermatológicas são as mais freqüentes, podendo representar o único sinal clínico da doença (KOUTINAS et al., 1993). FERRER et al. (1988) propuseram quatro padrões dermatológicos para a leishmaniose visceral canina (LV): padrão alopécicodescamativo, ulcerativo, nodular e pustular estéril. Outros sinais cutâneos freqüentemente encontrados são hiperqueratose nasodigital e onicogrifose (KOUTINAS et al., 1993). Na Europa o padrão dermatológicomaiscomuméodescamativo, podendo os cães com este padrão evoluírem para resolução clínica completa espontaneamente (KOUTINAS et al., 1999; PAPADOGIANNAKIS et al., 2005). O achado histopatológico predominante nas lesões tegumentares é a inflamação granulomatosa e/ou piogranulomatosa com variável número de formas amastigotas (SLAPPENDEL; FERRER, 1998; GROSS et al., 2005). Os eosinófilos e mastócitos são escassos e ocasionalmente células gigantes multinucleadas são observadas (GROSS et al., 2005). Em um estudo realizado na Grécia, vinte e dois cães com LV, foram avaliados quanto aos padrões histopatológicos de vários tipos de lesões tegumentares, e a perifoliculite granulomatosa foi a mais observada. As formas amastigotas, neste mesmo estudo, foram observadas em pouco menos de $50 \%$ das amostras coradas pela hematoxilina e eosina (KOUTINAS et al., 1993).

O objetivo deste trabalho foi avaliar as lesões macro e microscópicas da pele de cães com LV, da região de Araçatuba, estado de São Paulo, Brasil, com os padrões dermatológicos descamativos e ulcerativos.

\section{Material e Métodos}

Foram utilizados trinta cães, provenientes do Centro de Controle de Zoonoses do município de Araçatuba, região endêmica para a leishmaniose visceral. A confirmação do diagnóstico foi realizada empregando-se esfregaços de punção de linfonodo 
poplíteo (MOREIRA et al., 2002), corados pelo Diff-Quick $^{\circledR}$, e pela reação de imunofluorescência indireta (IFI) de amostras de soro obtidas do sangue coletado por punção veno-jugular, realizado pelo Laboratório de Sanidade Animal, da Universidade Federal do Piauí.

Os animais com diagnóstico confirmado foram divididos em dois grupos: cães com padrão dermatológico descamativo $(n=15)$ e outro com padrão dermatológico ulcerativo $(n=15)$. Os cães incluídos nos dois grupos não apresentavam sinais de alteração sistêmica da doença.

Fragmentos de pele foram colhidos de áreas com descamação e ulceração, após a eutanásia dos animais. Tal procedimento é uma norma para o controle da LVA no Brasil (BRASIL, 2003) e foi aprovado pelo comitê de Ética e Experimentação Animal da Faculdade de Medicina Veterinária e Zootecnia - Unesp, Botucatu, São Paulo. Após a coleta, as amostras foram fixadas em formol a $10 \%$ e processadas rotineiramente para histologia, com coloração pela hematoxilina e eosina (HE). A coloração pelo ácido periódico de Schiff (PAS) foi realizada para pesquisa de dermatófitos em todos os casos, sendo descartados os casos que foram observadas estruturas fúngicas (artroconídeos). As diferentes lesões histológicas encontradas na pele foram descritas de acordo com a média de percepção subjetiva de dois dos autores.

\section{Resultados}

Ao exame citopatológico, por punção aspirativa de linfonodo, foram observadas formas amastigotas em todos os casos $(n=30)$. Na reação de imunofluorescência indireta (IFD), as amostras resultaram acima do ponto de corte positivo (1:40) (EVANS et al., 1990). Os títulos sorológicos obtidos pela IFI foram 1:80 (2/30), 1:160 (5/30), 1:320 (14/30), 1:640 (5/30) e 1:1280(4/30).

Os cães com padrão dermatológico descamativo $(\mathrm{n}=15)$ apresentavam distribuição generalizada com escamas secas do tipo micácea e furfurácea, de cor branca a prateada $(15 / 15,100 \%)$ (Figura 1A). Os animais que apresentavam áreas hipotricóticas ou alopécicas tinham distribuição simétrica em regiões de pavilhão auricular e periocular $(13 / 15,86,6 \%)$. Demais lesões encontradas nos cães com dermatite descamativa foram: onicogrifose $(6 / 15,40 \%)$ e hiperqueratose em plano nasal $(6 / 15,40 \%)$.

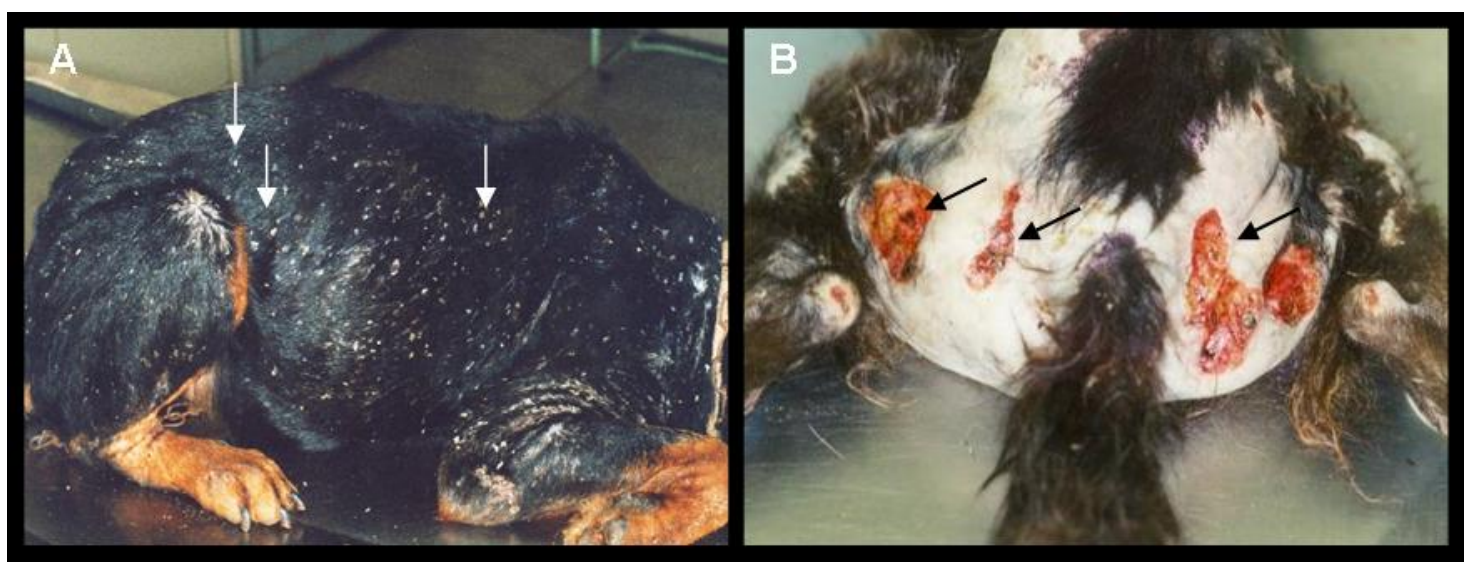

Figura 1. Fotografia de lesões macroscópicas de cães com Leishmaniose. (A) Dermatite descatimativa generalizada. Intensa descamação seca (setas) na região do tronco. (B) Dermatite ulcerativa. Presença de quatro grandes úlceras na região lombo-sacra (setas), este animal foi submetido a tricotomia para evidenciação das lesões. 
Todos os cães com padrão dermatológico ulcerativo apresentavam úlceras na região úmerorádio-ulnar $(15 / 15,100 \%)$, região do calcâneo $(13 / 15,86,6 \%)$, tuberosidade isquiática $(12 / 15$, $80 \%)$, superfície metatarso-tarsiana $(60 \% 9 / 15)$, pavilhão auricular $(3 / 15,20 \%)$, superfície metacarpo-carpiana $(2 / 15,13,3 \%)$ e regiões da narina, face, lábio, periocular, tóraco-ventral e lombossacra $(1 / 15,6,6 \%)$ (Figura 1B). Três cães deste grupo apresentavam discreta descamação furfurácea. Alopecia não foi observada neste grupo, inclusive na região periocular. Onicogrifose foi observada em sete cães $(46,6 \%)$.

Ao exame histopatológico do grupo com dermatite descamativa observou-se infiltrado inflamatório granulomatoso $(13 / 15,86,6 \%)$ (Figura $2 \mathrm{C})$ e piogranulomatoso $(2 / 15,13,3 \%)$ (Figura 2D), já nos cães com dermatite ulcerativa foi observado infiltrado piogranulomatoso $(8 / 15,53,3 \%)$ e granulomatoso $(7 / 15,46,6 \%)$.

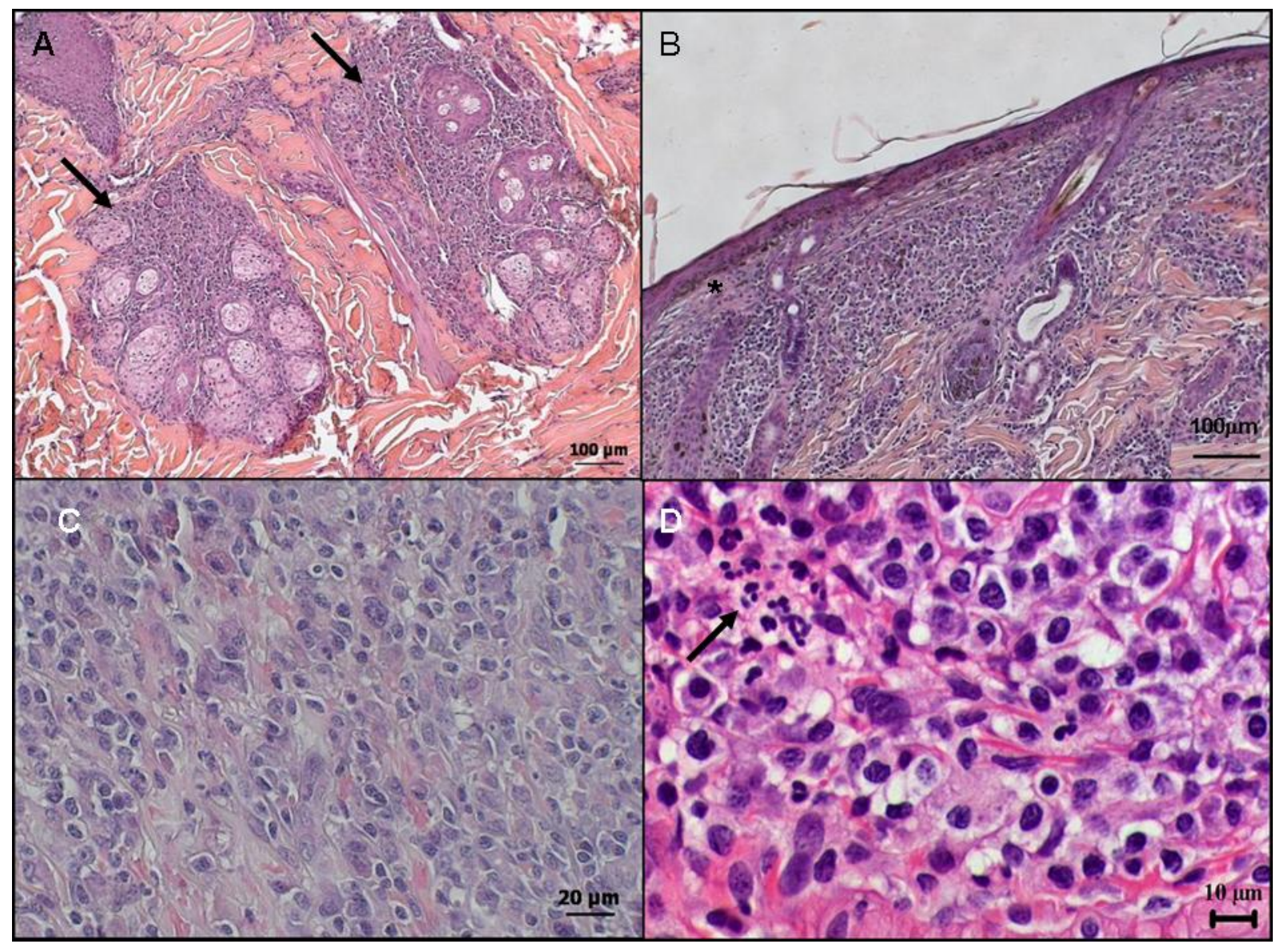

Figura 2. Exame Histopatológico. H\&E. Fotomicrografia das lesões de cães com Leishamaniose, apresentando Padrão Dermatológico Descamativo. (A) Dermatite Perianexial. Infiltrado inflamatório ao redor de anexos cutâneos (setas). (B) Dermatite liquenóide. Infiltrado inflamatório distribuído em faixa na interface epidermo-dermal (*). (C) Dermatite granulomatosa. Infiltrado inflamatório composto por macrófagos epitelióides, linfócitos e plasmócitos. (D) Dermatite piogranulomatosa. Infiltrado inflamatório composto por macrófagos epitelióides, linfócitos, plasmócitos e neutrófilos (seta). 
Os padrões histopatológicos encontrados nos cães com descamação foram: dermatite perianexial $(5 / 15,33,3 \%)$ (Figura 2A), dermatite perivascular superficial $(1 / 15,6,6 \%)$, dermatite nodular $(1 / 15$, $6,6 \%$ ), e as dermatites mistas, onde houve associação de dois padrões histopatológicos no mesmo corte $(8 / 15,53,3 \%)$, sendo, dermatite intersticial/ perianexial $(1 / 8,12,5 \%)$, dermatite liquenóide/ perivascular superficial e profunda $(1 / 8,12,5 \%)$ (Figura 2B), dermatite perivascular superficial e profunda/perianexial $(1 / 8,12,5 \%)$ e dermatite perivascular superficial/perianexial $(5 / 8,62,5 \%)$.

Nos cães com descamação as alterações histológicas epidérmicas foram: ortoqueratose $(8 / 15, \quad 53,3 \%)$, paraqueratose $(5 / 15,33,3 \%)$, hiperplasia $(7 / 15,46,6 \%)$, espongiose (2/15, $13,3 \%)$, exocitose de células mononucleares $(2 / 15,13,3 \%)$, melanose $(12 / 15,80 \%)$, crosta serofibrinoleucocitária $(5 / 15,33,3 \%)$ e serosa $(2 / 15,13,3 \%)$. As alterações glandulares foram: infiltrado inflamatório histiolinfoplasmocitário perisebáceo $(12 / 15,80 \%)$, e peri-sudoríparo (13/15, $86,6 \%$ ), com um cão apresentando hidradenite piogranulomatosa. As alterações foliculares foram: perifoliculite granulomatosa (13/15, 86,6\%), furunculose $(3 / 15,20 \%)$, dilatação infundibular $(6 / 15,40 \%)$, rolhões córneos infundibulares $(4 / 15$, $26,6 \%$ ), incontinência do pigmento melânico perifolicular $(8 / 15,53,3 \%)$. As alterações da derme incluíram vasculite leucocitoclástica $(2 / 15,13,3 \%)$, fibrose $(2 / 15,13,3 \%)$ e incontinência do pigmento melânico em derme superficial (6/15, 40\%). A presença de formas amastigotas foi observada em 53,3\% dos cães (Figura 3B).

Os padrões histopatológicos nos cães com lesões ulcerativas foram: dermatite perivascular superficial e profunda $(5 / 15,33,3 \%)$, dermatite difusa $(3 / 15$, $20 \%$ ) (Figura 2A), dermatite perianexial $(2 / 15$, $13,3 \%)$, dermatite nodular $(1 / 15,6,6 \%)$, e as mistas $(4 / 15,26,6 \%)$, representadas por dermatite intersticial/perivascular superficial e profunda $(1 / 4,25 \%)$, dermatite nodular/perianexial $(1 / 4$, $25 \%)$, dermatite fibrosante/perianexial $(1 / 4,25 \%)$ e dermatite perivascular superficial e profunda/ perianexial $(1 / 4,25 \%)$.

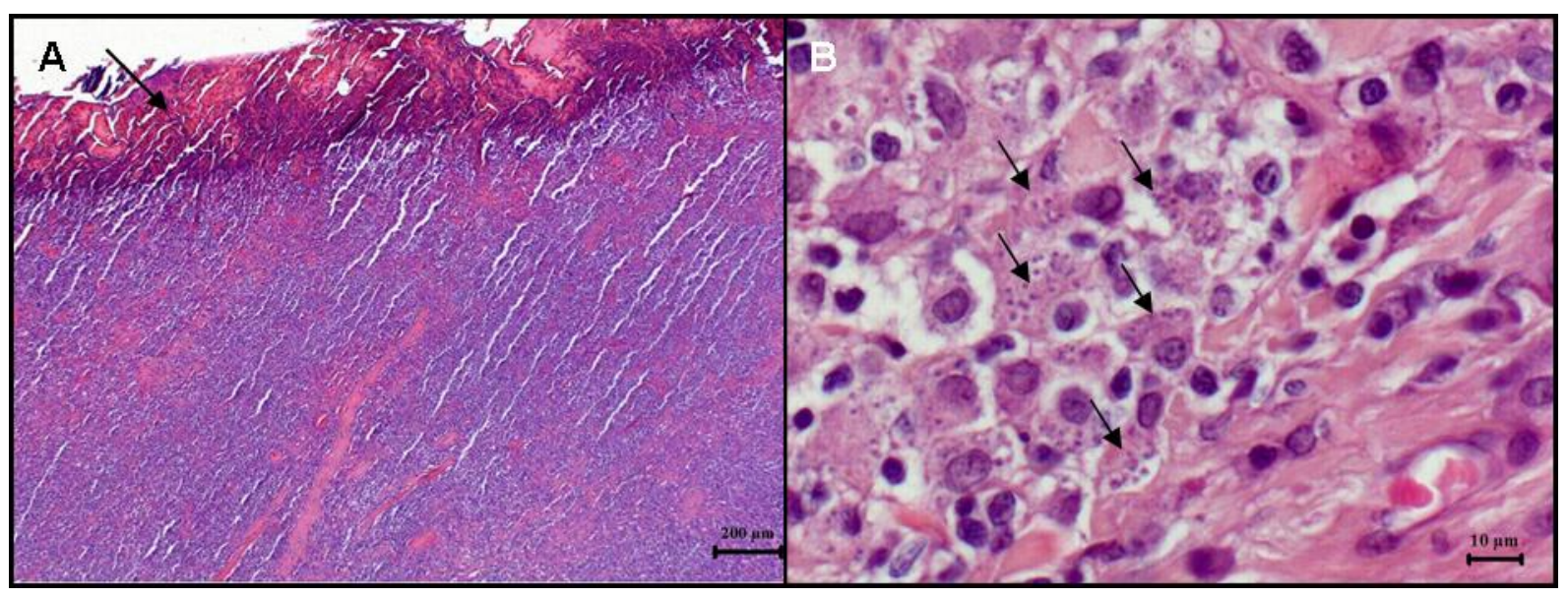

Figura 3. Exame Histopatológico. H\&E. Fotomicrografia das lesões de cães com Leishamaniose, apresentando Padrão Dermatológico Ulcerativo. (A) Dermatite difusa. Infiltrado inflamatório em difuso em derme, encimado por extensa úlcera (seta). (B) Formas amastigotas presentes no citoplasma de macrófagos (setas). 
Nos cães com DU, pela presença da ulceração $(15 / 15,100 \%)$, somente foram observadas como lesões epidérmicas, crostas serofibrinoleucocitária $(11 / 15,73,3 \%)$ e a presença de estruturas cocóides em colônias $(4 / 15,26,6 \%)$. As alterações glandulares foram: infiltrado inflamatório histiolinfoplasmocitário peri-sebáceo $(5 / 15,33,3 \%)$ e peri-sudoríparo $(11 / 15,73,3 \%)$. As alterações foliculares foram: perifoliculite granulomatosa $(6 / 15$, $40 \%)$ e furunculose $(4 / 15,26,6 \%)$. As alterações da derme incluiam vasculite leucocitoclástica $(2 / 15,13,3 \%)$, fibroplasia $(2 / 15,13,3 \%)$ e fibrose $(3 / 15,20 \%)$. A presença de formas amastigotas foi observada em $46,6 \%$ dos cães (Tabelas 1 e 2 ).

Tabela 1. Achados histopatológicos de lesões descamativas e ulcerativas de cães com Leishmaniose.

\begin{tabular}{lcc}
\hline \multicolumn{1}{c}{ lesões histopatológicas } & $\begin{array}{c}\text { Grupo Dermatite } \\
\text { Descamativa } \\
\mathrm{n}=15\end{array}$ & $\begin{array}{c}\text { Grupo Dermatite } \\
\text { Ulcerativa } \\
\mathrm{n}=15\end{array}$ \\
\hline ortoqueratose & $8 / 15(53,3 \%)$ & - \\
paraquratose & $5 / 15(33,3 \%)$ & - \\
hiperplasia & $7 / 15(46,6 \%)$ & - \\
espongiose & $2 / 15(13,3 \%)$ & - \\
exocitose & $2 / 15(13,3 \%)$ & - \\
melanose & $12 / 15(80 \%)$ & - \\
crosta serofibrinoleucocitária & $5 / 15(33,3 \%)$ & $11 / 15(73,3 \%)$ \\
crosta serosa & $2 / 15(13,3 \%)$ & - \\
adenite sebácea & $12 / 15(80 \%)$ & $5 / 15(33,3 \%)$ \\
adenite sudorípara & $13 / 15(86,6 \%)$ & $11 / 15(73,3 \%)$ \\
perifoliculite & $13 / 15(86,6 \%)$ & $6 / 15(40 \%)$ \\
furunculose & $3 / 15(20 \%)$ & $4 / 15(26,6 \%)$ \\
dilatação infundibular & $6 / 15(40 \%)$ & - \\
rolhões córneos & $4 / 15(26,6 \%)$ & - \\
incontinência pigmentar peri-folicular & $8 / 15(53,3 \%)$ & - \\
incontinência do pigmentar dérmica & $6 / 15(40 \%)$ & - \\
fibroplasia & - & $2 / 15(13,3 \%)$ \\
fibrose & $2 / 15(13,3 \%)$ & $3 / 15(20 \%)$ \\
vasculite leucocitoclástica & $2 / 15(13,3 \%)$ & $2 / 15(13,3 \%)$ \\
\hline
\end{tabular}

Tabela 2. Padrões histopatológicos das dermatites descamativas e ulcerativas da pele em cães com leishmaniose

\begin{tabular}{lcc}
\hline \multicolumn{1}{c}{ padrões histopatológicos } & $\begin{array}{c}\text { Grupo Dermatite } \\
\text { Descamativa } \\
\mathrm{n}=15\end{array}$ & $\begin{array}{c}\text { Grupo Dermatite Ulcerativa } \\
\mathrm{n}=15\end{array}$ \\
\hline dermatite perianexial & $5 / 15(33,3 \%)$ & $2 / 15(13,3 \%)$ \\
dermatite perivascular superficial & $1 / 15(6,6 \%)$ & - \\
dermatite nodular & $1 / 15(6,6 \%)$ & $1 / 15(6,6 \%)$ \\
dermatite nodular/perianexial & - & $1 / 4(25 \%)$ \\
dermatite intersticial/perianexial & $1 / 8(12,5 \%)$ & $5 / 15(33,3 \%)$ \\
dermatite liquenenóide/pvasc. sup. e prof. & $1 / 8(12,5 \%)$ & $1 / 4(25 \%)$ \\
dermatite pvasc. sup. e prof/perianexial & $1 / 8(12,5 \%)$ & - \\
dermatite perivascular superficial/perianexial & $5 / 8(62,5 \%)$ & $3 / 15(20 \%)$ \\
dermatite difusa & - & $1 / 4(25 \%)$ \\
dermatite intersticial/ pvasc. sup. e prof. & - & $1 / 4(25 \%)$ \\
dermatite fibrosante/perianexial & - & \\
\hline
\end{tabular}

pvasc. sup. e prof.: perivascular superficial e profundo 


\section{Discussão}

A seleção dos dois grupos se baseou nos trabalhos de FERRER et al. (1988) e KOUTINAS et al. (1993) nos quais as dermatites descamativas e ulcerativas foram as alterações mais encontradas nos cães com leishmaniose visceral. Os padrões nodular, pustular estéril (FERRER et al., 1988) e papular (ORDEIX et al., 2005) não foram observados durante o período de seleção dos animais. Nos cães com dermatite descamativa as escamas apresentavam distribuição generalizada do tipo seca, furfurácea ou micácea, de quantidade moderada a severa. A alopecia periocular bilateral, um aspecto dermatológico interessante, foi observada em nosso estudo com freqüência, como relatado na Europa (FERRER et al., 1988; KOUTINAS et al. 1993, CIARAMELLA et al., 1997) e em outros estudo no Brasil (SANTA ROSA; OLIVEIRA, 1997; FEITOSA et al., 2000).

Os cães com dermatite descamativa, histologicamente, apresentavam glândulas sebáceas destruídas ou com ductos obliterados por processo inflamatório, o que pode ter contribuído para uma queda na qualidade lipídica epidérmica, com conseqüente excessiva esfoliação cutânea (KOUTINAS et al. 1993; PAPADOGIANNAKIS et al. 2005). No entanto, esta não pode ser a única explicação para o quadro descamativo, pois três cães não apresentavam evidência de adenite sebácea. Talvez a alteração da epidermopoiese influenciada pelas citocinas linfo-histiocitárias possa ser um dos mecanismos que tenham contribuído para a descamação (SUTER et al. 1997).

Nos cães com úlceras, estas eram multifocais e apresentavam semelhanças com os aspectos ulcerativos da leishmaniose tegumentar americana, causada Leishmania (Viannia) brasiliensis (MADEIRA et al., 2004). As ulcerações eram circunscritas, anulares ou policíclicas, com bordas elevadas e fundo granuloso de cor vermelhovivo, recobertas ou não por exsudato seroso e serossanguinolento. Porém, as bordas infiltrativas, presentes no homem, que dão um aspecto chamado "em moldura de quadro" não foram observadas em nossos casos (SAMPAIO; RIVITTI, 2001).

É importante lembrar que na patogênese das lesões na leishmaniose tegumentar americana, o processo inflamatório e as formas amastigotas ficam confinados às lesões cutâneas ou mucocutânea, e não se disseminam, ao contrário da variante visceral nos cães, onde as lesões não se restringem à pele (SLAPPENDEL; FERRER, 1998; MADEIRA et al., 2004). Ao contrário do observado por Ferrer et al. (1988), no presente estudo, nenhum cão apresentava áreas de alopecia, quando categorizados neste padrão dermatológico.

A onicogrifose foi observada em pouco menos da metade dos cães nos dois grupos (seis cães com dermatite descamativa e sete com dermatite ulcerativa). Tal achado pode ser devido a matriz ungueal ser estimulada pela presença do parasito (NOLI, 1999).

Ao exame histopatológico, as lesões descamativas se caracterizaram por infiltrado inflamatório constituído por macrófagos epitelióides, poucos linfócitos e variável número de plasmócitos. Em dois casos os neútrofilos estavam presentes em grande quantidade conferindo um processo piogranulomatoso. Nas amostras descamativas os eosinófilos e mastócitos eram escassos.

A análise pelos padrões histopatológicos inflamatórios da pele é uma leitura baseada na arquitetura morfológica da lesão e não etiológica da enfermidade (ACKERMAN, 1978). Ela implica na leitura da lâmina com uma objetiva de pequeno aumento procurando localizar se o processo patológico está situado na epiderme, na derme, hipoderme, ou em alguma combinação destas (ACKERMAN, 1978). Considerando tal metodologia diagnóstica, diferentes alterações foram encontradas nas lesões descamativas e ulcerativas. Nos cães com dermatite descamativa os padrões histopatológicos mistos, foram os mais observados, seguidos da dermatite perianexial, perivascular superficial e nodular. Os padrões que coexistem em 
uma mesma lâmina histológica podem representar a evolução da leishmaniose ou sua associação com outra dermatose (KOUTINAS et al., 1993; GROSS et al., 2005).

Nos cães dos dois grupos a dermatite perianexial foi um achado freqüente, como o único padrão histopatológico ou associado a outros. O infiltrado inflamatório perianexial concentrava-se mais ao redor das glândulas sebáceas, quando estas estavam presentes, do que dos folículos pilosos e, em alguns casos, havia destruição glandular com restos de material sebáceo em meio ao infiltrado granulomatoso. Esta é uma característica presente na adenite sebácea granulomatosa idiopática, que acomete Poodles, Akitas e Vislas, sendo esta dermatose um diagnóstico diferencial importante quando não são observadas as formas amastigotas (GROSS et al., 2005; PAPADOGIANNAKIS et al., 2005).

O envolvimento granulomatoso do infiltrado nas glândulas sebáceas, nestes casos, deve ser cuidadosamente avaliado, sendo que a presença de numerosos histióciotos epitelióides, sugere mais se tratar de leishmaniose (KOUTINAS et al., 1993). Perifoliculite granulomatosa foi observado em treze cães, quase a totalidade dos cães com dermatite descamativa. Esteachadotambémfoifreqüentemente observado em outros estudos (FERRER et al. 1988; KOUTINAS et al., 1993; PAPADOGIANNAKIS et al. 2005). Esta distribuição do infiltrado inflamatório pode ser confundida com lesões granulomatosas orientadas perianexialmente que ocorrem na síndrome do granuloma-piogranuloma estéril canino (GROSS et al. 2005). Em um estudo sobre esta síndrome, mais da metade dos casos foram positivos para Leishmania sp, utilizando técnicas de biologia molecular (CORNEGLIANI et al., 2005).

A dermatite nodular nos cães decorre freqüentemente de quadro prévio de furunculose (YAGER; SCOTT, 1993), no entanto, não foi observado evidência de ruptura folicular nos casos de dermatite nodular em nosso estudo. O padrão nodular nestes casos pode ser decorrente da infecção por leishmania, pois nestas amostras foram observadas formas amastigotas em meio ao infiltrado nodular. Na reação liquenóide observada, esta não era caracterizada por degeneração hidrópica da camada basal e presença de corpos apoptóticos (Civatte), o que poderia sugerir alteração autoimune (GROSS et al. 2005).

Ferrer et al. (1988) relataram ser difícil a observação de formas amastigotas ao exame histológico de lesões ulcerativas. Em nosso estudo foi possível a identificação de formas amastigotas, redondas a ovais, dentro dos macrófagos ou extracelularmente, que se assemelhavam a diminutos "plasmócitos", em aproximadamente 50\% dos animais nos dois grupos.

As lesões descamativas nos cães com LV são caracterizadas pela expressão do complexo de histocompatibilidade maior classe II nas células de Langerhans e queratinócitos, e pela presença de um infiltrado inflamatório com predomínio de células $\mathrm{T}$ CD4+, e baixo número de formas amastigotas, sendo que este tipo lesional pode ter um bom prognóstico, evoluindo até para a cura espontânea (FONDEVILA et al., 1997; PAPADOGIANNAKIS et al., 2005).

As úlceras desenvolvidas em cães com leishmaniose pode seruma conseqüência da liberação do parasito dos macrófagos pela derme levando a uma intensa inflamação e vasculite (RIDLEY; RIDLEY, 1983). Esta vasculite, mesmo não vista histologicamente, pode corroborar na ocorrência das úlceras, como conseqüência de isquemia tecidual e de pressão em pontos de apoio. A presença de imunocomplexos circulantes, decorrente da infecção pelo protozoário pode ter contribuído para a presença de vasculite leucocitoclástica (NICHOLS et al., 2001).

Dermatite difusa, onde agregados celulares e a morfologia normal da derme não são visualizadas, foi observada em três cães com dermtite ulcerativa e em nenhum com dermatite descamativa, a solução de continuidade presente nessas lesões, levando 
a infecção secundária, corrobora para quadros inflamatórios mais intensos e um padrão difuso de distribuição do infiltrado celular.

Uma das principais causas de dermatite fibrosante nos cães é a furunculose (YAGER; SCOTT, 1993) e no grupo de cães com ulcerações foi observado este padrão juntamente com evidências de ruptura folicular.

\section{Conclusões}

As lesões descamativas, assim como as ulcerativas, dos cães com leishmaniose apresentam diferentes padrões de distribuição do infiltrado inflamatório, sendo que a ausência das formas amastigotas nas amostras histopatológicas dificulta o diagnóstico da doença. Nos padrões histopatológicos das lesões descamativas e ulcerativas dos cães deve-se incluir a leishamaniose como diagnóstico diferencial. Os achados dermatopatológicos encontrados neste levantamento assemelham-se aos da literatura internacional. No entanto, estes são dados nacionais inéditos que contribuem para o auxílio diagnóstico desta enfermidade infecciosa de grande importância para a saúde publica no Brasil.

\section{Agradecimento}

Ao Laboratório de Sanidade Animal, da Universidade Federal do Piauí e FUNDUNESP

\section{Referências}

ACKERMAN, A. B. Histologic diagnosis of inflamatory skin diseases: a method by pattern analysis. Philadelphia: Lea \& Febiger, 1978.

BRASIL. Ministério da Saúde. Manual de vigilância e controle da leishmaniose visceral. Brasília: Ministério da Saúde, 2003.

CIARAMELLA, P.; OLIVA, G.; DE LUNA, R.; GRADONI, L.; AMBROSIO, R.; CORTESE, L.; SCALONE, A.; PERSECHINO, A. A retrospective clinical study of canine leishmaniasis in 150 dogs naturally infected by Leishmania infantum. The Veterinary record, London, v. 141, n. 21, p. 539-543, 1997.
CORNEGLIANI, L.; FONDEVILA, D.; VERCELLI, A.; MANTERO, G.; FONDATI, A. PCR technique detection of Leishmania spp. but not Mycobacterium spp. in canine cutaneous 'sterile' pyogranuloma/ granuloma syndrome. Veterinary Dermatology, Oxford, v. 16, n. 4, p. 233-238, 2005.

EVANS, T. G.; VASCONCELOS, I. A. B.; LIMA, J. W. O.; TEIXEIRA, M. J.; MCAULLIFE, I. T.; LOPES, U. G.; PEARSON, R. D.; VASCONCELOS, A. W. Canine visceral leishmaniasis in northeast Brasil: Assessment of serodiagnostic methods. American Journal of Tropical Medicine and Hygiene, Mclean, v. 42, n. 2, p. 118-123, 1990.

FEITOSA, M. M.; IKEDA, F. A.; LUVIZOTTO, M. C. R.; PERRI, S. H. V. Aspectos clínicos de cães com leishamniose visceral no município de Araçatuba - São Paulo (Brasil). Clínica Veterinária, São Paulo, v. 28, n. 1, p. 36-44, 2000.

FERRER, L.; RABANAL, R.; FONDEVILA, D.; RAMOS, J. A.; DOMINGO, M. Skin lesion in canine leishmaniasis. Journal of Small Animal Practice, Oxford, v. 29, p. $381-388,1988$

FONDEVILA, D.; VILAFRANCA M.; FERRER L. Epidermal immunocompetence in canine leishmaniasis. Veterinay Immunology and Immunopathology. Amsterdam, v.56, p. 319-27, 1997.

GROSS, T. L.; IHRKE, P. J.; WALDER, E. J.; AFFOLTER, V. R. Skin diseases of the dog and cat: clinical and histopathologic diagnosis. Oxford: Blackwell, 2005.

KOUTINAS, A. F.; POLIZOPOULOU, Z. S.; SARIDOMICHELAKIS, M. N.; ARGYRIADIS, D.; FYTIANOU, A.; PLEVRAKI, K. G. Clinical considerations on canine visceral leishmaniasis in Greece: A retrospective study of 158 cases (1989-1996). Journal of the American Animal Hospital Association, Lakewood, v. 35, n. 5, p. 376-383, 1999.

KOUTINAS, A. F.; SCOTT, D. W.; KONTOS, V.; LEKKAS, S. Skin lesions in canine leishmaniasis (KalaAzar): a clinical and histopathological study on 22 spontaneous cases in Greece. Veterinary Dermatology, Oxford, v. 3, n. 3, p. 121-130, 1993.

LAISON, R.; SHAW, J. J. Ecology and epidemiology: new world. In: PETERS, W., KILLICK-KENDRICK, R. (Eds.). The leishmaniasis in biology and medicine. London: Academic Press, 1987. v.1, p.291-363.

MADEIRA, M. F.; SHUBACH, A. O.; SHUBACH, T. M. P.; LEAL, C. A; MARZOCHI, M. C. A. Identification of Leishmania (Leishmania) chagasi isolated from health skin of symptomatic and asymptomatic dogs seropositive 
for leishmaniasis in the municipality of Rio de Janeiro. The Brazilian Journal of Infectious Diseases, Salvador, v. 8, n. 6, p. 440-444, 2004.

MOREIRA, M. A. B.; LUVIZOTTO, M. C. R.; NUNES, C. M.; SILVA, T. C. C.; LAURENTI, M. D.; CORBERTT, C. E. P. Application of direct immunofluorescence technic for diagnosis of canine visceral leishmaniasis in lymph nodes aspirates. Brazilian Journal of Veterinary Research and Animal Science, São Paulo, v. 39, n. 1, p. 103-106, 2002.

NICHOLS, P. R.; MORRIS, D. O.; BEALE K. M. A retrospective study of canine and feline cutaneous vasculitis. Veterinary Dermatology, Oxford, v. 12, n. 5, p. 255-264, 2001.

NOLI, C. Leishmaniosis canina. Waltham Focus, London, v. 9, n. 2, p. 16-24, 1999.

ORDEIX, L.; SOLANO-GALLEGO, L.; FONDEVILA, D.; FERRER, L.; FONDATI, A. Papular dermatitis due to Leishmania spp. infection in dogs with parasite-specific cellular immune responses. Veterinary Dermatology, Oxford, v. 16, n. 3, p. 187-191, 2005.

PAPADOGIANNAKIS, E. I.; KOUTINAS, A. F.; SARIDOMICHELAKIS, M.; VLEMMAST, J.; LEKKAS, S.; KARAMERIS, A.; FYTIANOU, A. Cellular immunophenotyping of exfoliative dermatitis in canine leishmaniosis (Leishmania infantum). Veterinary Immunology and Immunopathology, Amsterdam, v. 104, n. 3-4, p. 227-237, 2005.
RIDLEY, D. S.; RIDLEY, M. The evolution of lesions in cutaneous leishmaniasis. Journal of Pathology, Sussex, v. 141, n. 1, p. 83-96, 1983.

SAMPAIO, S.; RIVITTI, E. A. Dermatologia. 2.ed. São Paulo: Artes Médicas, 2001.

SANTA ROSA, I. C. A.; OLIVEIRA, I. C. S. Leishmaniose visceral: breve revisão sobre uma zoonose reemergente. Clínica Veterinária, São Paulo, v. 11, n. 1, p. 24-28, 1997.

SANTOS, S. O.; ARIAS, J.; RIBEIRO, A. A.; HOFFMANN, M. P.; FREITAS, R. A.; MALACCO, M. A. F. Incrimination of Lutzomya cruzi as a vector of American visceral leishmaniasis. Medical and Veterinary Entomology, Oxford, v. 12, n. 3, p. 315-317, 1998.

SLAPPENDEL, R. J.; FERRER, L. Leishmaniasis. In: GREENE, C. E. (Ed.). Infectious diseases of the dog and cat. Philadelphia: Saunders, 1998. p. 450-458.

SUTER, M. M.; CRAMERI, F. M.; OLIVRY, T.; MUELLER, E.; VON TSCHARNER, C.; JENSEN, P. J. Keratinocyte biology and pathology. Veterinary Dermatology, Oxford, v. 8, n. 1, p. 67-100, 1997.

YAGER, A. J.; SCOTT, D. W. The skin and appendages. In: JUBB, K. V. F.; KENNEDY, P. C.; PALMER, N. Pathology of domestic animals. 4.ed. San Diego: Academic Press, 1993. p. 531-738. 